\title{
Model of Metamaterial Based on Graphene Scrolls and Carbon Nanotubes with Negative Refractive Index ${ }^{1}$
}

\author{
A. I. Siahlo ${ }^{a}$, N. A. Poklonski ${ }^{a, *}$, S. A. Vyrko ${ }^{a}$, and S. V. Ratkevich ${ }^{a}$ \\ ${ }^{a}$ Physics Department, Belarusian State University, Minsk, 220030 Belarus \\ *e-mail:poklonski@bsu.by
}

\begin{abstract}
The structure of a new metamaterial with negative permittivity and permeability that is made of periodically positioned graphene/boron nitride nanoscrolls and carbon nanotubes is proposed. The parameters of the metamaterial structure with a negative refractive index for the frequency of an electromagnetic wave within the visible range (namely, from near infrared to yellow) are calculated.
\end{abstract}

DOI: $10.1134 / \mathrm{S} 1063782618140294$

\section{INTRODUCTION}

Optical properties of artificial periodic structures with negative values for both permittivity $\varepsilon=\varepsilon_{r} \varepsilon_{0}$ and permeability $\mu=\mu_{r} \mu_{0}\left(\varepsilon_{r}<0\right.$ and $\mu_{r}<0$ are the real parts of relative permittivity and permeability, $\varepsilon_{0}$ and $\mu_{0}$ are the electric and magnetic constants) were considered in detail in [1,2].

The dispersion equation $k^{2}=\left(\omega^{2} / c^{2}\right) n^{2}$, where $\omega$ is the angular frequency, $c=\left(\varepsilon_{0} \mu_{0}\right)^{-1 / 2}$ is the speed of light in vacuum, $n$ is the refractive index that relates the values of the wave vector $k$ and the angular frequency $\omega$ of the electromagnetic wave does not change its form if both $\varepsilon_{r}$ and $\mu_{r}$ have negative values [1]. However, while for light propagation in vacuum $\left(\varepsilon_{r}=\mu_{r}=1\right)$ or in ordinary materials $\left(\varepsilon_{r}>0\right.$ and $\left.\mu_{r}>0\right)$ the vectors of electric $\mathbf{E}$ and magnetic $\mathbf{H}$ components of the electromagnetic field together with the wave vector $\mathbf{k}=k \mathbf{n}_{\mathbf{k}}$ (where $\mathbf{n}_{\mathbf{k}}$ is the unit vector directed along $\mathbf{k}$ ) form a right-handed system of vectors, for $\varepsilon_{r}<0$ and $\mu_{r}<0$ the system of vectors $(\mathbf{E}, \mathbf{H}, \mathbf{k})$ is a left-handed one. Artificial periodic structures (metamaterials) for which both $\varepsilon_{r}<0$ and $\mu_{r}<0$ are called left-handed materials [2].

Positive electromagnetic wave energy in the metamaterial is achieved only if its phase velocity $\mathbf{v}=\mathbf{n}_{\mathbf{k}} \omega / k$ and group velocity $\mathbf{v}_{\mathrm{gr}}=d \omega / d \mathbf{k}$ have opposite directions, thus the metamaterial can be characterized by the negative refractive index $n=-\sqrt{\varepsilon_{r} \mu_{r}}<0$. An important effect observed in the metamaterials is the change in the refraction law at the interface of an ordinary material and a metamaterial (see Fig. 1). At the incidence of an electromagnetic wave on the interface of a right-handed and a left-handed material the

\footnotetext{
${ }^{1}$ The article is published in the original.
}

transmitted wave is on the same side from the normal to the interface as the incident wave [1] (Fig. 1).

In an isotropic medium, the solution of the system of Maxwell's equations [3, 4] for the projections of the components of a plane electromagnetic wave that vary with time $t$ in a Cartesian coordinate system $(x, y, z)$,

$$
\begin{gathered}
-\partial H_{y} / \partial z=\varepsilon \partial E_{x} / \partial t, \\
\partial E_{x} / \partial z=-\mu \partial H_{y} / \partial t
\end{gathered}
$$

can be written in the form:

$$
\begin{gathered}
E_{x}=E_{0 x} \exp \left[-i\left(k_{z} z-\omega t\right)\right], \\
H_{y}=H_{0 y} \exp \left[-i\left(k_{z} z-\omega t\right)\right],
\end{gathered}
$$

where $i=\sqrt{-1}$.

If both $\varepsilon$ and $\mu$ are positive, then the vectors $\mathbf{E}, \mathbf{H}$ and $\mathbf{k}$ form a right-handed system of vectors. If the value of either $\varepsilon$ or $\mu$ in the medium is positive and the value of the other quantity is negative, then the solu-

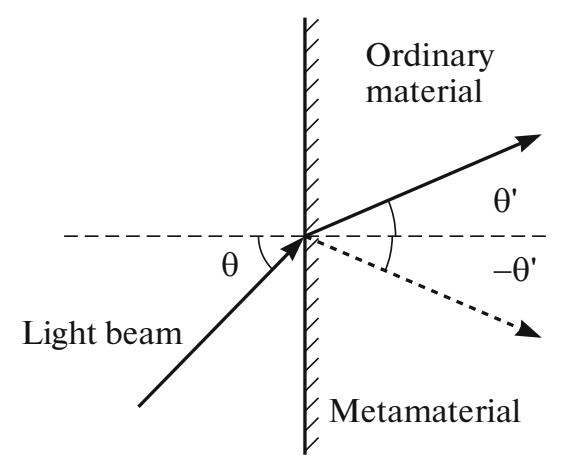

Fig. 1. Refraction of the light beam incident at the angle $\theta$ to the normal at the interface of an ordinary material and a metamaterial. 
tion of Eq. (1) can be written in the form of Eq. (2), only if either $k_{z}$ or $\omega$ are imaginary quantities. In such a medium the electromagnetic wave is damped. If the values of both $\varepsilon$ and $\mu$ are negative, then the solution of Eq. (1) can be written in the form of Eq. (2), however with either $k_{z}<0$ or $E_{0 x}<0$ or $H_{0 y}<0$, which is fulfilled if the system of vectors $\mathbf{E}, \mathbf{H}$ and $\mathbf{k}$ is a lefthanded one.

Metamaterials with negative permeability $\mu<0$ in the electromagnetic field are considered in papers $[2,5]$ and can be a system of rolls of a conductive material (SRCs-"Swiss roll" capacitors). The direct current in each roll cannot flow around it, however there is a considerable capacitance in the roll that enables high-frequency alternating current to flow. The effective permeability of a system of SRCs is given by the expression [5]:

$$
\mu_{r}=1-\frac{\pi r^{2}}{a^{2}}\left(1-\frac{c^{2} d}{2(N-1) \pi^{2} \omega^{2} r^{3}}\right)^{-1},
$$

where $a$ is the distance between the centers of neighbor rolls, $r$ is the outer radius of the roll, $d$ is the spacing between the turns of the roll, $N$ is the number of turns in the roll. In a medium of SRCs, in a frequency range determined by the geometric parameters of the system, for a magnetic field oriented along the rolls there will be a negative permeability $\mu<0$.

Negative permittivity $\varepsilon_{r}$ exists in the ordinary metal. The behavior of the electron gas in the metal is well-described by the Drude model [6,7] with relative permittivity

$$
\varepsilon_{\mathrm{D}}=1-\frac{\omega_{p}^{2}}{\omega^{2}+i \omega \gamma},
$$

where $\omega_{p}$ is the plasma frequency, $\gamma$ is the frequency of collisions with scattering centers (attenuation parameter) of delocalized electrons; $\gamma \approx 5 \times 10^{13} \mathrm{rad} / \mathrm{s}$ for silver; $\varepsilon_{r}=\operatorname{Re}\left(\varepsilon_{\mathrm{D}}\right)=\left(\omega^{2}-\omega_{p}^{2}+\gamma^{2}\right) /\left(\omega^{2}+\gamma^{2}\right)$. From Eq. (4) for $\gamma \rightarrow 0$ it follows that $\varepsilon_{\mathrm{D}}=1-\left(\omega_{p} / \omega\right)^{2}$. Thus, below the plasma frequency $\omega_{p}$ the permittivity $\varepsilon_{r}$ is negative, and the value of the plasma frequency $\omega_{p}$ is proportional to the square root of the concentration of delocalized electrons.

It is shown in [8], that in the medium made of periodically positioned small metal rods the dependence $\varepsilon(\omega)$ is described by the Drude-like model (see Eq. (4)) with the "plasma frequency"

$$
\omega_{p}^{2}=\frac{2 \pi c^{2}}{a^{2} \ln (a / R)},
$$

where $a$ is the distance between the rods and $2 R$ is the rod diameter. [In the derivation of Eq. (5), the deciding factor was the self-induction of the structure made of the rods.]
In such a medium, as in a bulk metal, the permittivity $\varepsilon_{r}$ (more precisely, the $\varepsilon_{r}$ component in the direction of the rod axis) will be negative in a certain frequency range $\left(\omega<\omega_{p}\right)$. Note that the "plasma frequency" $\omega_{p}$ depends on geometric parameters, hence the value $\varepsilon_{r}$ can be controlled by changing the frequency $\omega_{p}$ by varying the length-to-radius ratio of the rods.

Metamaterials, for which both $\varepsilon_{r}$ and $\mu_{r}$ are negative, can be used for controlling the propagation of electromagnetic waves: in particular for creating objects that do not reflect or absorb electromagnetic radiation [9] and for focusing electromagnetic radiation [2].

Metamaterials for which both permittivity and permeability are negative $\left(\varepsilon_{r}<0\right.$ and $\left.\mu_{r}<0\right)$ based on nanocomposites made of multiwalled carbon nanotubes (MWCNT) combined with polyaniline (PANI) were studied in [10].

The purpose of this work is to simulate a new type of metamaterial made of graphene and boron nitride bilayer nanoscrolls [11] combined with a network of parallel conducting armchair $(m, m)$ carbon nanotubes positioned perpendicular to nanoscrolls.

The graphene layers of the nanoscroll are the conducting layers and the boron nitride layers are the insulating layers between the conducting layers. Both the bilayer nanoscrolls (with the outer radius $R_{\text {out }}$ ) and the nanotubes (with the diameter $d_{(m, m)}$ ) are positioned with a period $a=2 R_{\text {out }}+d_{(m, m)}+2 h$, where $h=$ $0.335 \mathrm{~nm}$ is the minimum distance between the carbon or boron nitride layers ( $h$ is also the thickness of the graphene layer).

\section{RESULTS AND DISCUSSION}

To form a material with a negative magnetic permeability in the desired frequency range we propose using graphene/boron nitride bilayer nanoscrolls formed from bilayer ribbons of $120 \mathrm{~nm}$ in length positioned with a period of $a$.

In accordance with the model [11], a bilayer nanoscroll with the spacing between the turns $d=2 h=$ $2 \cdot 0.335 \mathrm{~nm}=0.67 \mathrm{~nm}$, made of heterostructure graphene/boron nitride nanoribbons of length $L=$ $120 \mathrm{~nm}$, in a steady state has internal and outer radii $R_{\text {in }}=2.9 \mathrm{~nm}$ and $R_{\text {out }}=5.8 \mathrm{~nm}$; the number of turns $N=\left(\varphi_{\text {out }}-\varphi_{\text {in }}\right) / 2 \pi=4.4$ (Fig. 2 ).

The value of the negative magnetic permeability of a system of nanoscrolls is given by Eq. (3). Calculations based on Eq. (3) neglect the resistivity of the nanoscroll material $(\rho=0)$. 


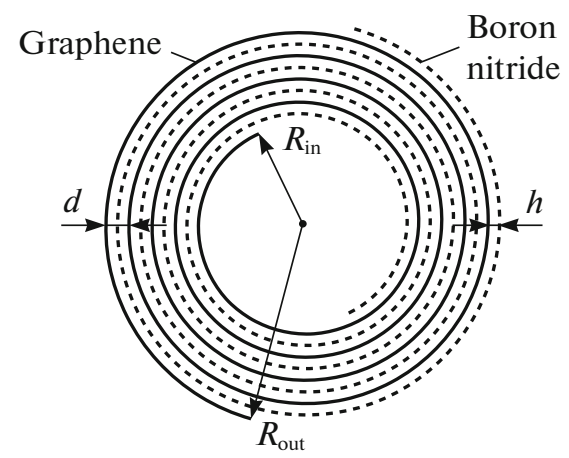

Fig. 2. Bilayer nanoscroll made of graphene/boron nitride ribbons of length $L=120 \mathrm{~nm}$ in a steady state.

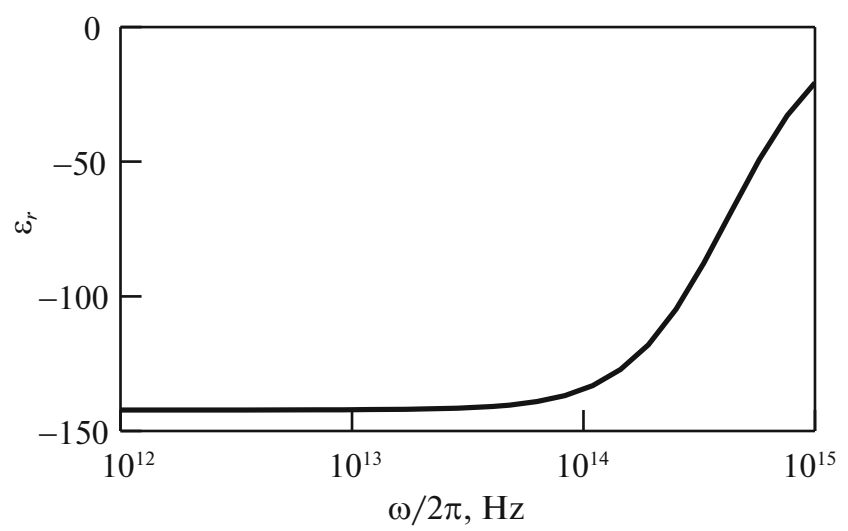

Fig. 3. Dependence of the real component of the permittivity on the frequency for a material made of nanotubes $(10,10)$ positioned with a period of $a=13.7 \mathrm{~nm}$.

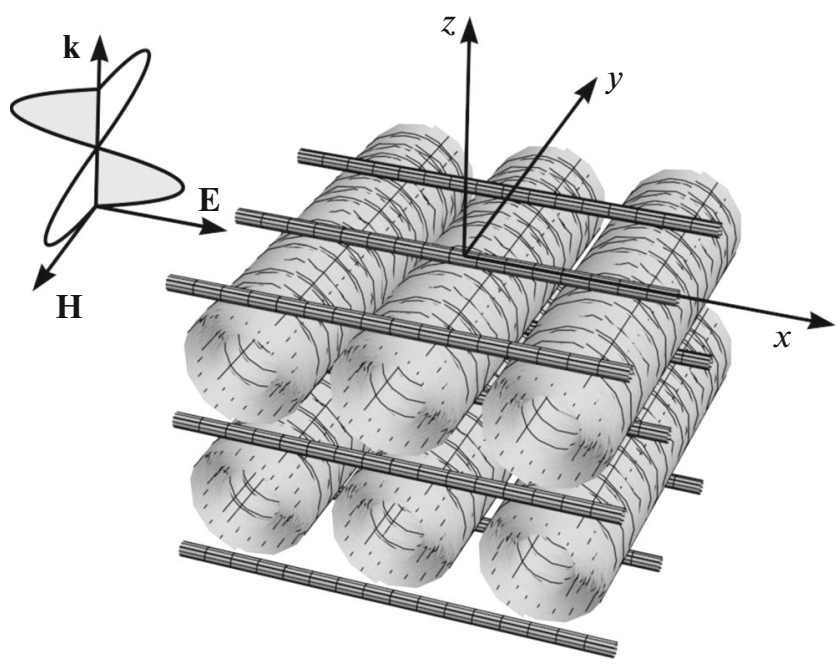

Fig. 4. Structure of the metamaterial made of carbon nanoscrolls directed along the $y$ axis and carbon nanotubes directed along the $x$ axis and possible orientation of the vectors $\mathbf{E}, \mathbf{H}$ and $\mathbf{k}$ for propagation of a plane electromagnetic wave in such square lattice metamaterial with a period of $a=13.7 \mathrm{~nm}$ (E directed along the carbon nanotubes; $|\mathbf{k}| a \ll 1)$.
In accordance with Eq. (3), the angular frequencies $\omega_{\mu}^{\infty}\left(\right.$ at which $\mu_{r} \rightarrow \infty$ ) and $\omega_{\mu}^{0}$ (at which $\mu_{r} \rightarrow 0$ ) are equal to

$$
\begin{gathered}
\omega_{\mu}^{\infty}=\frac{c}{\pi r^{3 / 2} \sqrt{\frac{d}{2(N-1)}}} ; \\
\omega_{\mu}^{0}=\frac{c}{\pi r^{3 / 2}} \sqrt{\frac{d}{2(N-1)\left(1-\pi r^{2} / a^{2}\right)} .}
\end{gathered}
$$

For the structure under consideration made of nanoscrolls with $r=R_{\text {out }}=5.8 \mathrm{~nm}, d=0.67 \mathrm{~nm}, N=$ 4.4 , positioned at the distance $a=13.7 \mathrm{~nm}>2 R_{\text {out }}$ we get $f_{\mu \infty}=\omega_{\mu}^{\infty} / 2 \pi=3.4 \times 10^{14} \mathrm{~Hz}$ and $f_{\mu 0}=\omega_{\mu}^{0} / 2 \pi=$ $5.2 \times 10^{14} \mathrm{~Hz}$ (from near infrared to yellow).

Let us consider the possibility of forming a material from a network of periodically positioned nanotubes that has a negative real component of the permittivity $\left(\varepsilon_{r}<0\right)$ in the frequency range corresponding to the visible electromagnetic radiation. Let this network consists of conductive armchair $(10,10)$ nanotubes, the diameter of each nanotube is $2 R=d_{(10,10)}=$ $10 \times 3 a_{\mathrm{CC}} / \pi=1.36 \mathrm{~nm}$, where $a_{\mathrm{CC}}=0.142 \mathrm{~nm}$ is the distance between the nearest carbon atoms in the nanotube. Nanotubes are positioned at the distance $a=2 R_{\text {out }}+d_{(10,10)}+2 h=13.7 \mathrm{~nm}$ relative to each other. The plasma angular frequency $\omega_{p}$ for such a system of parallel nanotubes, calculated from Eq. (5), is equal to $\omega_{p}=3.2 \times 10^{16} \mathrm{rad} / \mathrm{s}$.

The calculation of the parameter $\gamma$ (analogue to the inverse of the relaxation time or attenuation parameter) for the nanotube in Eq. (4) is carried out according to the formula [8]:

$$
\gamma=\frac{\varepsilon_{0} a^{2} \omega_{p}^{2}}{\pi R^{2} \sigma}
$$

where $\sigma$ is the dc electrical conductivity of the nanotube. It is known [12] that the maximum conductivity of a single-walled carbon nanotube is determined by the conduction quantum $G_{0}=7.75 \times 10^{-5} \mathrm{Ohm}^{-1}$. In accordance with the estimations of [13], we assume that the electrical conductivity of each nanotube (longer than $1 \mu \mathrm{m}$ ), of which the system is composed, is equal to $\sigma=4.3 \times 10^{8} \mathrm{Ohm}^{-1} \mathrm{~m}^{-1}$, which is 7 times higher than the electrical conductivity of silver. It follows from Eq. (6) that for the proposed system of nanotubes $\gamma=2.7 \times 10^{15} \mathrm{~s}^{-1}$. Note that the electrical conductivity of graphene and of single-walled carbon nanotubes can be controlled by passivation with hydrogen $[14,15]$ or by filling with metal atoms [16].

The real values of the permittivity $\varepsilon_{r}=\operatorname{Re}\left(\varepsilon_{\mathrm{D}}\right)$ calculated from Eq. (4) are shown in Fig. 3. In the frequency range from $10^{12}$ to $10^{15} \mathrm{~Hz}$, including frequencies $\omega / 2 \pi$ of visible radiation (from $3.8 \times 10^{14}$ to $7.8 \times$ $10^{14} \mathrm{~Hz}$ ), the values of $\varepsilon_{r}$ are negative and vary within the limits of $-142<\varepsilon_{r}<-20$. 


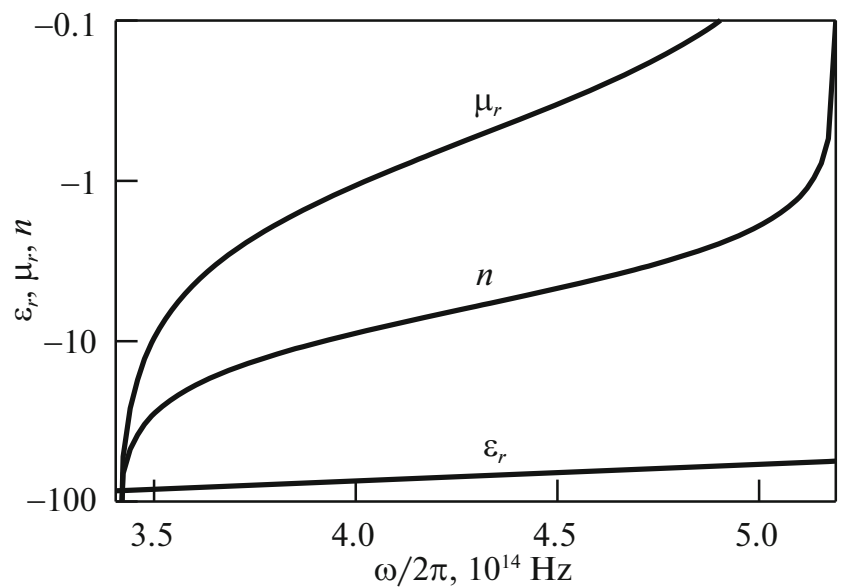

Fig. 5. Calculated relative permittivity $\varepsilon_{r}$, relative permeability $\mu_{r}$, and refractive index $n$ of the metamaterial in the frequency range $\omega / 2 \pi$ from $3.4 \times 10^{14}$ to $5.2 \times 10^{14} \mathrm{~Hz}$ (from near infrared to yellow).

Let us consider a material made of nanoscrolls [11] combined with a network of parallel carbon nanotubes, oriented perpendicular to the nanoscrolls (see Fig. 4). A network of conducting nanotubes provides a Drude-like dependence of the $\varepsilon_{r}$ of the metamaterial on $\omega / 2 \pi$ for the electric component $\mathbf{E}$ directed along the nanotube axes [8], while the graphene/boron nitride bilayer nanoscrolls allow to obtain negative values of permeability $\mu_{r}$ for given frequencies.

A calculation according to the models $[5,8]$ was performed using Eqs. (3) and (4) for a metamaterial composed of "metallic" $(10,10)$ single-walled carbon nanotubes of diameter $d_{(10,10)}=1.36 \mathrm{~nm}$ positioned in a square lattice with a period $a=2 R_{\text {out }}+d_{(10,10)}+2 h=$ $13.7 \mathrm{~nm}$, and bilayer graphene/boron nitride nanoscrolls with internal $R_{\text {in }}=2.9 \mathrm{~nm}$ and outer $R_{\text {out }}=$ $5.8 \mathrm{~nm}$ radii positioned perpendicular to the nanotubes in a square lattice with the same period $a$. This metamaterial provides changes in the values of $\varepsilon_{r}, \mu_{r}$, and $n=$ $-\sqrt{\varepsilon_{r} \mu_{r}}$ within the ranges of $-86<\varepsilon_{r}<-56,-100<$ $\mu_{r}<0$, and $-100<n<0$ for the frequency $\omega / 2 \pi$ range from $3.4 \times 10^{14}$ to $5.2 \times 10^{14} \mathrm{~Hz}$ (Fig. 5). For the frequency of $3.8 \times 10^{14} \mathrm{~Hz}$ (the beginning of the visible frequency band), $\varepsilon_{r}=-78, \mu_{r}=-1.9$, and $n=-12$.

\section{CONCLUSIONS}

A new metamaterial with negative permittivity $\varepsilon_{r}$ and permeability $\mu_{r}$ made of periodically positioned graphene/boron nitride nanoscrolls and carbon nanotubes is proposed. The parameters of the metamaterial structure with the negative refractive index $n<0$ for the visible light (from near infrared to yellow) are calculated. For the beginning of the visible frequency band $\left(3.8 \times 10^{14} \mathrm{~Hz}\right) \varepsilon_{r}=-78, \mu_{r}=-1.9$, and $n=-12$.

\section{ACKNOWLEDGMENTS}

The work has been supported in part by the Belarusian Republican Foundation for Fundamental Research (grant no. F18R-253) and Belarusian National Research Program "Convergence-2020".

\section{REFERENCES}

1. V. G. Veselago, Sov. Phys. Usp. 10, 509 (1968).

2. I. B. Vendik and O. G. Vendik, Tech. Phys. 58, 1 (2013).

3. M. A. Remnev and V. V. Klimov, Phys. Usp. 61, 157 (2018).

4. H. Haus, Waves and Fields in Optoelectronics (PrenticeHall, Englewood Cliffs, NJ, 1984).

5. J. B. Pendry, A. J. Holden, D. J. Robbins, and W. J. Stewart, IEEE Trans. Microwave Theory Technol. 47, 2075 (1999).

6. V. I. Balykin and P. N. Melentiev, Phys. Usp. 61, 133 (2018).

7. M. Grundmann, The Physics of Semiconductors. An Introduction Including Nanophysics and Applications (Springer, Berlin, 2016).

8. J. B. Pendry, A. J. Holden, W. J. Stewart, and I. Youngs, Phys. Rev. Lett. 76, 4773 (1996).

9. J. B. Pendry, Y. Luo, and R. Zhao, Science (Washington, DC, U. S.) 348, 521 (2015).

10. X. Yao, X. Kou, and J. Qiu, Carbon 107, 261 (2016).

11. A. I. Siahlo, N. A. Poklonski, A. V. Lebedev, I. V. Lebedeva, A. M. Popov, S. A. Vyrko, A. A. Knizhnik, and Yu. E. Lozovik, Phys. Rev. Mater. 2, 036001 (2018).

12. R. Saito, G. Dresselhaus, and M. S. Dresselhaus, Physical Properties of Carbon Nanotubes (Imperial College Press, London, 1998).

13. A. V. Eletskii, Phys. Usp. 52, 209 (2009).

14. L. A. Chernozatonskii, P. B. Sorokin, and A. A. Artukh, Russ. Chem. Rev. 83, 251 (2014).

15. A. H. Castro Neto, F. Guinea, N. M. R. Peres, K. S. Novoselov, and A. K. Geim, Rev. Mod. Phys. 81, 109 (2009).

16. N. A. Poklonskii, E. F. Kislyakov, G. G. Fedoruk, and S. A. Vyrko, Phys. Solid State 42, 1966 (2000). 